Treatment completion rates rose $(82 \%-90 \%)$. The proportion of cases lost to follow-up reduced $(2.5 \%-0 \%)$. The proportion of smear positive pulmonary cases with at least one risk factor on Directly Observed Therapy increased from $42 \%$ to $67 \%$. Uptake of HIV testing rose $(71 \%-89 \%)$. The proportion of pulmonary TB cases with at least 1 and $>5$ contacts identified both increased $(64 \%-84 \%$ and $50 \%-67 \%$, respectively).

In conclusion, within a short space of time cohort review has led to an improved index case management and contact tracing process in our service. It improves accountability, enhances patient management and facilitates staff education. Accurate, comprehensive and prompt data underpin this success. If this is sustained, we believe that cohort review will result in improved patient outcomes. It provides, also, an excellent structure within which advances such as the National Strain Typing project may be introduced to achieve maximal clinical impact.

\section{P61 \\ KAREL STYBLO COMES TO TOWN: STAFF PERSPECTIVES ON TB COHORT REVIEW}

doi:10.1136/thoraxjnl-2011-201054c.61

${ }^{1} \mathrm{C}$ Anderson, ${ }^{1} \mathrm{~S}$ Tamne, ${ }^{2} \mathrm{~J}$ White, ${ }^{1} \mathrm{I}$ Abubakar, ${ }^{1} \mathrm{~S}$ Anderson, ${ }^{2} \mathrm{~S}$ Dart, ${ }^{2} \mathrm{~J}$ DeKoningh, ${ }^{2} \mathrm{M}$ Lipman. ${ }^{1}$ Health Protection Agency, London, UK; ${ }^{2}$ Royal Free Hospital, London, UK

Since its creation by Dr Karel Styblo, the cohort review (CR) principle of systematically analysing treatment outcome of every notified TB case and contact investigation in a brief, timely, structured manner, has been implemented widely outside of the UK-with impressive results. In 2010, North Central London (NCL) TB service adopted the concept, trained staff members and put in practice CR. Given the resource implications that our service faced already, plus the potential for its introduction to result in an increased staff workload, we undertook a survey of how CR was perceived and the impact it had on those involved in its use.

After four rounds of CR (12 months from introduction), an anonymous on-line survey was sent to NCL staff members plus external participants and observers who had attended at least one review. The survey explored participant's personal and institutional response to the organisation, impact and outcome of CR's introduction. It was sent to 88 individuals. 72 ( $82 \%$ ) completed the 10 min online questionnaire.

Over $95 \%$ felt that CR identified gaps in service, most frequently: collaboration with other TB services (69\%), patient care $(63 \%)$, collaboration with allied services (51\%) and service organisation (45\%). Just over a third felt that CR highlighted training needs, especially for contact tracing. $70 \%$ reported changes to their way of working-in particular altering practice in response to apparent weaknesses in their approach to contact tracing. $86 \%$ felt that CR led to improvement in the speed of interventions, better data quality and enhanced professional relations. A small number of staff noted negative consequences which largely reflected increase in initial work load.

Cohort review has enhanced our service provision. It is well received by local staff as well as external participants \& observers. Our data are encouraging; and we hope will assist in promoting roll out to other parts of the country.

\section{P62 \\ DRUG INDUCED HYPOTHYROIDISM IN PATIENTS RECEIVING TREATMENT FOR MULTI-DRUG RESISTANT TUBERCULOSIS}

doi:10.1136/thoraxjn--2011-201054c.62

\begin{abstract}
1,2J Gupta, ${ }^{1,2} \mathrm{R}$ A M Breen, ${ }^{1,2} \mathrm{H}$ J Milburn. 'Guy's, King's and St Thomas' School of Medicine, London, UK; ${ }^{2}$ Department of Respiratory Medicine, Guy's and St Thomas' NHS Foundation Trust, London, UK
\end{abstract}

Introduction and Objectives Drug-induced hypothyroidism is an uncommon adverse effect of treatment for multi-drug resistant tuberculosis (MDR-TB), with a limited number of case reports from the 1950s reporting the issue. The most likely agents to cause hypothyroidism are $p$-aminosalicylic acid (PAS), and to a lesser extent Prothionamide, both commonly used in regimens to treat MDR-TB. We report five cases of MDR-TB, four of whom developed hypothyroidism while on treatment. This is the largest reported cohort to have developed drug-induced hypothyroidism as a result of MDR-TB treatment to date. We analysed if there were any predisposing or causative factors which may have contributed to patients developing hypothyroidism.

Method Patients were seen in clinic on a regular basis and possible adverse events evaluated by symptom review and clinical evaluation. Thyroid function tests (TFTs) were ordered based upon clinical suspicion. Following identification of hypothyroidism patients were started on thyroxine replacement therapy with monitoring of TFT levels to normalise them. Patient demographics were collected for analysis.

Results Four out of five patients ( 3 females and 1 male, aged 29-40 years) developed hypothyroidism following MDR-TB treatment with regimens containing PAS and Prothionamide. The dominant presenting symptom was lethargy, with one developing goitre and hair loss. All patients were from ethnic minorities born overseas in: India (1); Bangladesh (1) and Somalia (2). The $5^{\text {th }}$ female Nepalese patient remained euthyroid. Patients had been in the UK from 3 to 6 years with no travel history of note since. Hypothyroidism developed at varying stages of treatment from 101-442 days. On analysis of predisposing or causative factors for hypothyroidism development, those patients who originated from areas of iodine deficiency (eg, Bangladesh) developed hypothyroidism sooner after commencing treatment and took longer for euthyroid resolution despite receiving increased dosages of thyroxine replacement therapy.

Conclusion Individuals originating from areas of iodine deficiency have an increased likelihood of developing drug induced hypothyroidism when receiving a regimen containing PAS and/or Prothionamide for MDR-TB treatment. As symptoms of hypothyroidism were generally non-specific and could easily be ascribed to $\mathrm{TB}$, we suggest monitoring TFTs in all patients on prolonged treatment regimens containing PAS and/or Prothionamide.

\section{P63 THE PREVALENCE OF VIRAL HEPATITIS IN PATIENTS UNDERGOING ANTI-TUBERCULOUS THERAPY}

doi:10.1136/thoraxjnl-2011-201054c.63

${ }^{1} \mathrm{M}$ Asgheddi, ${ }^{2} \mathrm{D} \mathrm{W}$ Connell, ${ }^{1} \mathrm{H}$ A Nooredinvand, ${ }^{1} \mathrm{M}$ Abdullah, ${ }^{2} \mathrm{M}$ O'Donoghue ${ }^{1} \mathrm{~L}$ Campbell, ${ }^{2} \mathrm{~A}$ Lalvani, ${ }^{2} \mathrm{M}$ Wickremasinghe, ${ }^{1} \mathrm{~S}$ Khan, ${ }^{2} \mathrm{O} \mathrm{M}$ Kon. ${ }^{1}$ Hepatology and Gastroenterology Section, Division of Diabetes Endocrinology and Metabolism, Department of Medicine, Imperial College Healthcare NHS Trust, London, UK, ${ }^{2}$ Tuberculosis Service, Chest and Allergy Clinic, St Mary's Hospital, Imperial College Healthcare NHS Trust, London, UK

Background One-third of the world's population is thought to be infected with Mycobacterium tuberculosis (Mtb); the highest incidence rates for active disease are found in the WHO's South-East Asian and African regions. Hepatitis B virus (HBV) and Hepatitis C virus (HCV) each infect about $0.3 \%$ of the UK population. HBV/ $\mathrm{HCV}$ are treatable but largely asymptomatic until advanced liver disease and/or cancer. Screening for $\mathrm{HBV} / \mathrm{HCV}$ is recommended in high-risk individuals but is not routinely performed in TB patients. $\mathrm{HBV} / \mathrm{HCV}$ share similar epidemiological "hotspots" to TB, and several studies, primarily in South America and East Asia, have shown an increased prevalence of HBV/HCV in TB patients and an association between $\mathrm{HBV} / \mathrm{HCV}$ and Drug Induced Liver Injury 
(DILI) following anti-TB therapy. No such analysis has been performed in the UK.

Aims To assess

1. The prevalence of markers of $\mathrm{HBV}$ and $\mathrm{HCV}$ infection in patients undergoing anti-TB therapy.

2. Whether serological evidence of $\mathrm{HBV} / \mathrm{HCV}$ increases risk of DILI.

Method A prospective study on 429 TB patients receiving anti-TB therapy in a diverse urban TB clinic. Serological markers of HBV/ $\mathrm{HCV} / \mathrm{HIV}$ infection were documented. ALT was measured prior to treatment and 2-4 weeks after treatment initiation. DILI was defined as an ALT rise of greater than 2-times upper limit of normal following a normal pre-treatment level $(<40 \mathrm{IU} / \mathrm{ml})$.

Results $58.7 \%$ of patients were from either South-East Asia or Africa. $61(14.2 \%)$ patients were isolated anti-HBc antibody positive (Anti-HBc), $11(2.6 \%)$ were Hepatitis B surface antigen positive (HBsAg) and $7(1.6 \%)$ were HCV antibody positive. The prevalence of active $\mathrm{HBV} / \mathrm{HCV}$ infection was significantly higher than the estimated UK prevalence in this urban TB clinic. Three $(5.3 \%)$ patients with serological evidence of $\mathrm{HBV} / \mathrm{HCV}$ had DILI compared to $25(9.5 \%)$ of those without.

Conclusion HBV/HCV prevalence is higher among TB patients and routine screening for viral hepatitis should be considered in this group. DILI was not increased in patients with serological markers of $\mathrm{HBV} / \mathrm{HCV}$.

\section{P64 THE EFFECT OF WOOD SMOKE ON THE ABILITY OF HUMAN MACROPHAGES TO PHAGOCYTOSE AND KILL MYCOBACTERIUM TUBERCULOSIS}

doi:10.1136/thoraxjnl-2011-201054c.64

${ }^{1} \mathrm{~J}$ E Scriven, ${ }^{2} \mathrm{D}$ G Fullerton, ${ }^{1} \mathrm{~A}$ Warman, ${ }^{1} \mathrm{~S}$ B Gordon. ${ }^{1}$ Liverpool School of Tropical Medicine, Liverpool, UK; ${ }^{2}$ University Hospital Aintree, Liverpool, UK

Introduction Half of the world's population are exposed to household air pollution from biomass fuels, which are increasingly recognised as a global risk factor for impaired respiratory health. Data from case-control studies has associated Tuberculosis (TB) with biomass fuel exposure. We hypothesised that particulate matter (PM) within alveolar macrophages of individuals exposed to biomass smoke detrimentally affects macrophage function by: (A) Reduced phagocytosis of TB, leading to increased infectivity and (B) Impaired macrophage killing of $\mathrm{TB}$, leading to increased susceptibility to disease. This study assessed the ability of wood smoke exposed human monocyte-derived macrophages (MDMs) to phagocytose and kill TB in vitro.

Methods MDMs, from buffy coats, were cultured in vitro for 12 days and then exposed to suspensions of wood smoke PM (Malawian (MW) and Norwegian (NW) wood, $10 \mu \mathrm{g} / \mathrm{ml}$ or $50 \mu \mathrm{g} /$ $\mathrm{ml}$ ) for $8 \mathrm{~h}$. The PM loaded cells were then challenged with TB. Phagocytosis was assessed by manually counting infected macrophages, using fluorescent microscopy and by quantitative culture of day 1 supernatant and macrophage lysate. Remaining cells were maintained in culture for 7 days before lysis and quantitative culture to assess intracellular growth.

Results Microscopy data showed a reduced proportion of infected macrophages in the high dose NW smoke group (Abstract P64 figure $1 \mathrm{~A})$, and fewer TB bacilli per macrophage in the two MW smoke groups (Abstract P64 figure 1B). Quantitative culture of day 1 supernatant and cell lysate showed no difference between the groups (Abstract P64 figure 1C). Quantitative culture of day 7 lysate showed reduced Mycobacteria loads in the high dose NW smoke group (Abstract P64 figure 1D).
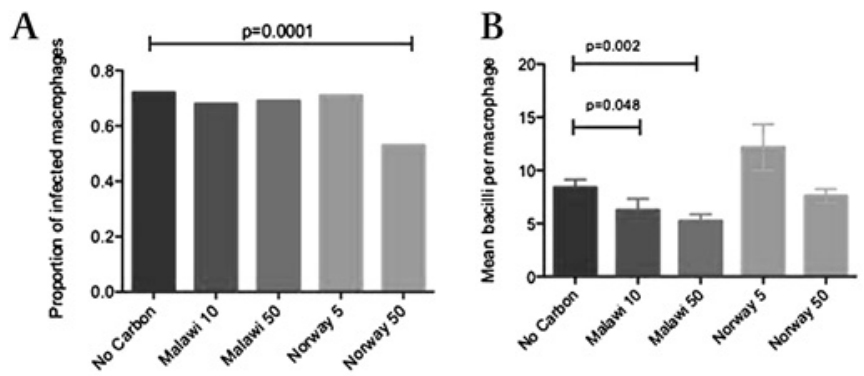

C
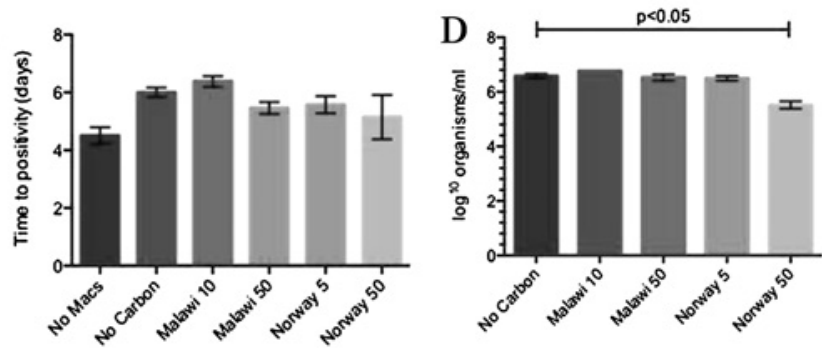

Abstract P64 Figure 1 The effect of wood smoke on monocyte-derived macrophages to phagocytose and kill Mycobacterium tuberculosis: (A) Effects of wood smoke on macrophage phagocytosis of TB (assessed by microscopy). (B) Mean numbers of bacilli per infected macrophage. (C) Mean time to positivity for culture of day 1 supernatants. (D) Calculated Mycobacterial load of day 7 lysates (log scale).

Discussion We have developed an in vitro model to assess the interaction between TB and wood smoke exposed macrophages. Phagocytic data does not fully support increased TB infectivity in biomass smoke exposed individuals. Quantitative culture data does not demonstrate a difference in the ability of wood smoke exposed MDMs to kill TB; high dose NW smoke led to a reduced intramacrophage growth of TB probably as a result of the toxic effect of PM on Mycobacteria growth. Modifications of this model are required to provide mechanistic evidence of an association of $\mathrm{TB}$ with wood smoke exposure.

\section{P65 TREATING TUBERCULOSIS IN RURAL SOUTH AFRICA} doi:10.1136/thoraxjnl-2011-201054c.65

J N Periselneris. Church of Scotland Hospital, Tugela Ferry, KwaZulu-Natal, South Africa

Methods All the patients admitted to the male and female Tuberculosis wards at a rural hospital in KwaZulu-Natal, South Africa, during a 1-month period were entered into a database. Basic demographic data were collected as well as information about HIV status, medications, complications and 1-month mortality. The area's tuberculosis (TB) incidence is 1046 per 100000 and there is an HIV infection rate of $29 \%$.

Results 70 patients were admitted during the time allocated. $89 \%$ were HIV positive. The mean CD4 count of the HIV positive patients was 114 , however only $45 \%$ of patients were on ARVs at time of admission. $21 \%$ of patients had extrapulmonary TB. Only $17 \%$ were AFB positive, a further $9 \%$ had culture results back, two patients cultured proven multidrug resistant TB. Two patients had a history of completed XDR TB treatment. 10\% patients developed drug induced hepatitis, $3 \%$ had pneumothoraces, $10 \%$ had coexistant renal impairment, $11 \%$ had neurological complications. There was a $23 \% 1$-month mortality. No patients who were HIV negative died. $27 \%$ died of respiratory failure, $12 \%$ had hepatic failure, likely secondary to antituberculous medication. 